\title{
Population growth of Mexican free-tailed bats (Tadarida brasiliensis mexicana) predates human agricultural activity
}

\author{
Amy L Russell ${ }^{1 *+}$, Murray P Cox ${ }^{2,3,4+}$, Veronica A Brown ${ }^{5}$ and Gary F McCracken ${ }^{5}$
}

\begin{abstract}
Background: Human activities, such as agriculture, hunting, and habitat modification, exert a significant effect on native species. Although many species have suffered population declines, increased population fragmentation, or even extinction in connection with these human impacts, others seem to have benefitted from human modification of their habitat. Here we examine whether population growth in an insectivorous bat (Tadarida brasiliensis mexicana) can be attributed to the widespread expansion of agriculture in North America following European settlement. Colonies of T. b. mexicana are extremely large $\left(\sim 10^{6}\right.$ individuals $)$ and, in the modern era, major agricultural insect pests form an important component of their food resource. It is thus hypothesized that the growth of these insectivorous bat populations was coupled to the expansion of agricultural land use in North America over the last few centuries.

Results: We sequenced one haploid and one autosomal locus to determine the rate and time of onset of population growth in T. b. mexicana. Using an approximate Maximum Likelihood method, we have determined that T. b. mexicana populations began to grow $\sim 220$ kya from a relatively small ancestral effective population size before reaching the large effective population size observed today.

Conclusions: Our analyses reject the hypothesis that T. b. mexicana populations grew in connection with the expansion of human agriculture in North America, and instead suggest that this growth commenced long before the arrival of humans. As T. brasiliensis is a subtropical species, we hypothesize that the observed signals of population growth may instead reflect range expansions of ancestral bat populations from southern glacial refugia during the tail end of the Pleistocene.
\end{abstract}

\section{Background}

Modern human populations and their activities have had a significant, and frequently negative, impact on other organisms [1-3]. Human populations exert a tremendous ecological and evolutionary pressure on native species, comparable in total effect to that of glaciations on temperate species but operating over much shorter timescales $\left(10^{1}-10^{3}\right.$ versus $10^{4}-10^{5}$ years; $\left.[4,5]\right)$. The effect of human activities on native species is not easily predictable, and in some instances human activities have benefited native wildlife, often through increasing habitat or

\footnotetext{
* Correspondence: russelam@gvsu.edu

† Contributed equally

'Department of Biology, Grand Valley State University, Allendale, MI 49401, USA

Full list of author information is available at the end of the article
}

food availability $[6,7]$. Genetic methods are starting to prove useful for linking demographic processes in animal species to human activity, with recent studies attributing decreasing effective population size and increasing population fragmentation to anthropogenic deforestation, the expansion of agriculture, road construction and the presence of human settlements [8-10]. Here, we use genetic analyses to investigate whether anthropogenic forces, specifically the spread of agriculture, or non-anthropogenic forces such as the retreat of the Laurentide and Cordilleran glaciers, have driven the population expansion of a North American bat.

Roosting colonies of Mexican free-tailed bats (Tadarida brasiliensis mexicana) are some of the largest and most conspicuous aggregations of bats in North America. While historic colony counts numbered in the tens
C Biomed Central

(c) 2011 Russell et al; licensee BioMed Central Ltd. This is an Open Access article distributed under the terms of the Creative Commons Attribution License (http://creativecommons.org/licenses/by/2.0), which permits unrestricted use, distribution, and reproduction in any medium, provided the original work is properly cited. 
of millions $[11,12]$, more recent, and likely more accurate, exit counts using thermal imaging and infrared tracking methods estimate the total census size of freetailed bat colonies throughout the entire southwestern United States at 9 million individuals [13]. However, when the southwestern United States and Mexico are considered together, the census population size of this species may easily reach $10^{7}-10^{8}$ individuals, making it one of the most numerous known non-human mammals. The largest known aggregations of Mexican freetailed bats are in nursery colonies, primarily hosting reproductive adult females and their young. During the energetically demanding period of pregnancy and lactation, females ingest up to two-thirds of their body weight in insects every night [14]. These colonies thus depend upon a large and reliable base of insect prey to maintain their considerable population sizes.

A number of studies have documented strong links between Mexican free-tailed bats and important agricultural pest insects, especially Helicoverpa zea and Spodoptera exigua (Lepidoptera: Noctuidae). The adults of these moth species migrate northwards in the spring from Mexico to the United States, often flying at high altitudes to take advantage of prevailing winds [15]. High-altitude echolocation surveys show that Tadarida feeding calls are coincident in time and altitude with these migrating insect populations [16]. Molecular analyses also document significant levels of $H$. zea and S. exigua DNA in the feces of Mexican free-tailed bats [17], thus further verifying this predator-prey relationship. Although the diet of Mexican free-tailed bats is not restricted to agricultural pest insects [18], the development of human agriculture likely resulted in predictable swarms of pest insects caused in part by increasing levels of plant cultivation. Mexican free-tailed bats now exploit this resource heavily, especially during pregnancy and lactation [19].

This predator-prey relationship between Mexican freetailed bats and agricultural pest insects suggests that we should observe population growth in bats coupled to increases in insect prey in connection with human agricultural practices in the Americas. Population growth would most likely be associated with the widespread expansion of European agriculture during the last few centuries, or as an outside possibility, with the emergence of Native American agriculture during the last few thousand years [20]. More concretely, population growth of Mexican free-tailed bats cannot be linked to anthropogenic processes if it occurred before humans arrived in the Americas around 9-15 thousand years ago (kya) [21].

Population growth leaves distinct patterns of variation in genetic data, and these patterns can be used to infer effective sizes and growth rates, as well as estimate the time at which growth commenced [22]. Genetic variation in $T . b$. mexicana is consistent with population growth; mismatch distribution analyses of mitochondrial DNA sequence data are suggestive of population expansion occurring in concert with the development of human agriculture (2.7-3.0 kya; [23]). However, these analyses lack statistical power to distinguish between anthropogenic and climatic drivers of population growth, and this particular test often exhibits high false positive rates [24]. In this paper we take advantage of more statistically rigorous methods to infer these demographic parameters and evaluate the anthropogenic influence hypothesis. Here, we explore the rate and time of population growth in Mexican free-tailed bats using one of these advanced inferential methods, approximate Maximum Likelihood. A quantitative analysis of the rate and timing of population growth in T. b. mexicana can help us understand the response of wildlife species to major innovations in human cultural evolution such as the development of agriculture.

\section{Results}

\section{Approximate maximum likelihood}

Summary statistics suggest that Mexican free-tailed bat populations have experienced growth (Table 1), as do published neutrality tests and mismatch distributions [23]. Genetic diversity is particularly high for the mtDNA locus $\left(\theta_{W}=0.068\right.$ per bp), whereas diversity for the $R A G 2$ gene is an order of magnitude lower $\left(\theta_{W}=\right.$ 0.0065 per $\mathrm{bp})$. However, this disparity primarily reflects differences in the mutation rates of these two loci (i.e., $2.0 \times 10^{-8}$ and $8.6 \times 10^{-10} / \mathrm{bp} /$ year, respectively). More tellingly, singleton polymorphisms - a signal of population growth - are frequent in both datasets, accounting for $40 \%$ of all polymorphisms in the mtDNA control region and $32 \%$ of all polymorphisms in the RAG2 locus. Reflecting these high levels of singletons, Tajima's $D$ is negative for both loci $(T D=-1.5$ for the control

\begin{tabular}{|c|c|c|c|}
\hline Summary Statistic & Symbol & $\begin{array}{c}\text { Control region } \\
(\mathrm{mtDNA})\end{array}$ & $\begin{array}{c}\text { RAG2 } \\
\text { (autosomal) }\end{array}$ \\
\hline $\begin{array}{l}\text { Sample size } \\
\text { (chromosomes) }\end{array}$ & & 94 & 150 \\
\hline Sequence length (bp) & & 474 & 686 \\
\hline Segregating sites & S & 154 & 25 \\
\hline $\begin{array}{l}\text { Watterson's theta (per } \\
\text { bp) }\end{array}$ & $\theta_{\mathrm{W}}$ & 0.068 & 0.0065 \\
\hline $\begin{array}{l}\text { Pairwise differences (per } \\
\text { bp) }\end{array}$ & $\theta_{\pi}$ & 0.038 & 0.0047 \\
\hline Tajima's D & TD & -1.5 & -0.82 \\
\hline Singletons & $\eta_{1}$ & 62 & 8 \\
\hline Haplotypes & h & 86 & 52 \\
\hline
\end{tabular}


region and $T D=-0.82$ for $R A G 2$ ). Although such values are broadly indicative of growth, more sophisticated analyses are necessary to statistically exclude the possibility that Mexican free-tailed bat populations have actually remained constant in size and, if such a model can be rejected, to infer the rate and time of onset of their growth.

We turned to approximate Maximum Likelihood to explore a two-phase population growth model (Figure 1). We applied this method to our empirical dataset, first, to the two loci individually, and subsequently, to the two loci together (Table 2). Considering the mtDNA control region alone, the Maximum Likelihood estimate (MLE) suggests an ancestral effective population size of $\sim 120$ thousand, a modern effective population size of $\sim 11$ million, and an onset of growth of $\sim 330$ kya (Additional file 1). Considering RAG2 alone, the MLE suggests an ancestral effective population size of $\sim 340$ thousand, a modern effective population size of $\sim 6$ million, and an onset of growth of $\sim 110$ kya (Additional file 2). Importantly, however, the actual demographic history that produced the observed data must be compatible with both loci taken together; thus, to determine the demography that best fits both of our genetic loci, we considered the combined likelihood for the mtDNA control region and autosomal RAG2 locus (Figure 2). Our combined MLE favors an ancestral effective population size of $\sim 230$ thousand, a modern effective population size of $\sim 12$ million, and an onset of growth $\sim 220 \mathrm{ka}$. Only 63 grid points (of 1000, i.e., 6.3\%) within the 3-dimensional parameter space of $N_{A}, N_{0}$, and $\tau$ are significantly likely (i.e., these points form the $95 \%$ confidence interval) (Additional file 3, points ranked by likelihood). This set includes ranges of $\sim 120-450$ thousand

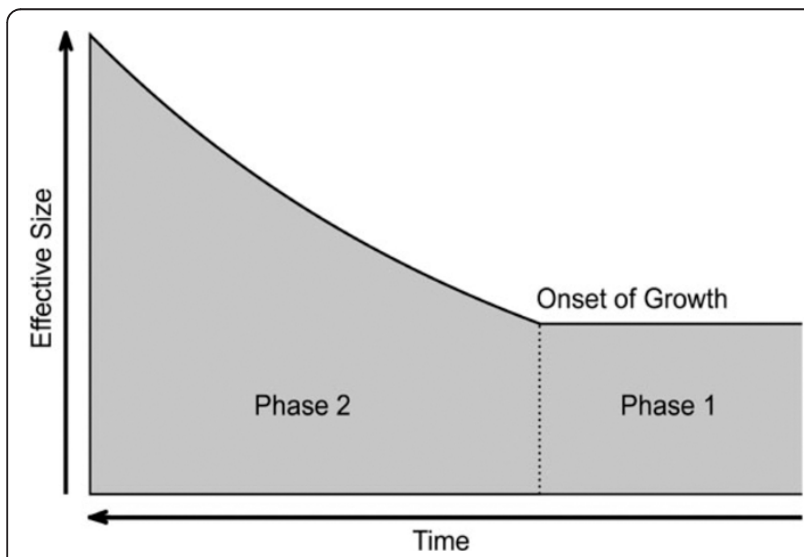

Figure 1 Two-phase model of population growth. An early period of constant size (Phase 1) is followed by a period of population growth (Phase 2). The dotted line reflects the time of onset of growth, during which the effective population size increases exponentially from ancestral to modern levels.
Table 2 Maximum likelihood estimates for modern and ancestral effective population sizes, time of onset of growth and population growth rates

\begin{tabular}{lcccccc}
\hline $\begin{array}{l}\text { Demographic } \\
\text { Parameter }\end{array}$ & \multicolumn{2}{c}{ mtDNA } & \multicolumn{2}{c}{ RAG2 } & \multicolumn{2}{c}{ Combined } \\
\cline { 2 - 7 } & MLE & $\mathbf{9 5 \% ~ C l}$ & MLE & $\mathbf{9 5 \% ~ C l}$ & MLE & $\mathbf{9 5 \% ~ C l}$ \\
\hline $\mathrm{N}_{\mathrm{A}}\left(\times 10^{3}\right)$ & 120 & $10-890$ & 340 & $120-560$ & 230 & $120-450$ \\
$\mathrm{~N}_{\mathrm{O}}\left(\times 10^{6}\right)$ & 11 & $6-50$ & 6 & $6-50$ & 11 & $6-50$ \\
$\tau(\mathrm{kya})$ & 330 & $110-500$ & 110 & $0-500$ & 220 & $110-500$ \\
$\alpha\left(\times 10^{-5} /\right.$ generation $)$ & 5.4 & $2.5-18$ & 10 & $2.6->>100$ & 7 & $2.6-18$ \\
Fold growth & 93 & $10-3900$ & 16 & $10-420$ & 48 & $16-320$ \\
\hline
\end{tabular}

for $N_{A}, \sim 6-50$ million for $N_{O}$, and $\sim 120-500$ kya for $\tau$. We note that the point representing constant effective size (i.e., no growth, $N_{O}=N_{A}$, or equivalently, $\alpha=0$ ) is rejected with strong statistical significance. In general, the ancestral effective size $N_{A}$ and the time of onset of growth $\tau$ were inferred with some certainty. The haploid locus contains more information about time (cf. Additional file 1), whereas the autosomal locus, with its larger effective size and thus older time to the most recent common ancestor (TMRCA), carries more information about the ancestral effective size (Additional file 2). We had less power to infer the modern effective size $N_{o}$, as is evident from the profile likelihood curve derived from the combined likelihood surface (i.e., generated for $N_{A}$, $N_{O}$ and $\tau$ separately; Additional file 4). However, this

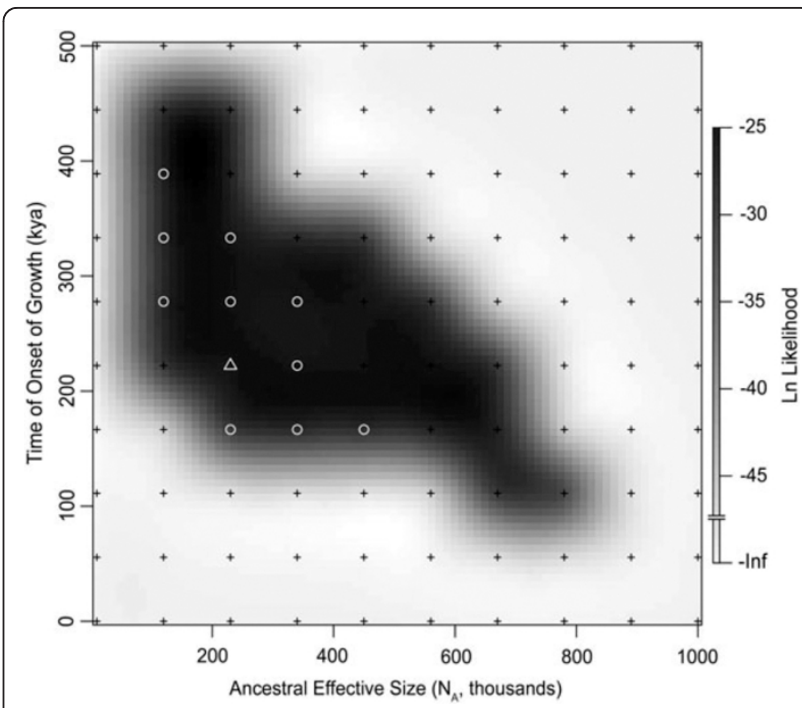

Figure 2 Combined log-likelihood surface $\left(N_{A}\right.$ versus $\left.\tau\right)$ for the haploid mtDNA control region and autosomal RAG2 locus. Black and white points indicate the grid of sampling locations. Loglikelihoods at these points are known with certainty, whereas loglikelihoods in the intervening spaces are interpolated. Regions of the parameter space with highest likelihood are shaded black. Only highlighted white points (circles and triangles) fall within the $95 \%$ confidence interval. The maximum likelihood estimate (MLE) is indicated by a white triangle. $N_{0}$ is set to its value for the MLE. 
uncertainty in $N_{O}$ is accommodated naturally by the likelihood function, and is thus accounted for in all the demographic values that we present here.

Note too that our three demographic parameters (i.e., $N_{A}, N_{O}$, and $\tau$ ) are not independent, but are instead correlated to various extents. Considering grid points within the $95 \%$ confidence interval of our combined likelihood surface (Additional file 3), the two effective sizes $\left(N_{A}\right.$ and $\left.N_{O}\right)$ are weakly correlated (Spearman's rank correlation; $r_{s}=0.32, P=0.01$ ). This explains $\sim 11 \%$ of the observed variance. However, the interdependence of the effective population sizes and the time of onset of growth is more pronounced. We find that $\tau$ is very strongly, negatively correlated with $N_{A}\left(r_{s}=\right.$ -0.68, $P<<0.0001)$ and $N_{O}\left(r_{s}=-0.76, P=0.0001\right)$, thus explaining $\sim 46 \%$ and $\sim 58 \%$ of the observed variance, respectively. Both associations are non-linear, and in each case, the time of onset of growth appears younger as the effective size increases. This suggests that development of new summary statistics with increased power to distinguish effective population sizes and/or the time of onset of growth would help to reduce the size of our $95 \%$ confidence interval by decoupling this parameter dependence.

\section{Validation}

Even though we used only standard methods, we still validated our inference technique using data simulated under known demographic histories. To do so, we generated coalescent trees and ancestral recombination graphs (ARG) at $10^{3}$ values of $N_{A}, N_{0}$, and $\tau$ drawn randomly from a uniform distribution across the parameter space, and calculated the values of $S, \eta_{1}$, and $h$ returned by each of these simulations. For each dataset, we then applied the approximate Maximum Likelihood algorithm described above, and calculated 95\% confidence intervals for $N_{A}, N_{O}$, and $\tau$. We considered individual test cases to be successful when our inferred demography included the known (i.e., defined) values of $N_{A}, N_{o}$, and $\tau$ within its 95\% confidence intervals. By setting the type I error rate at 0.05 , we would just by chance expect to infer $N_{A}, N_{O}$, and $\tau$ incorrectly for $\sim 5 \%$ of our simulated datasets. In practice, we observe a somewhat higher type I error rate of 9\% (i.e., a marginally enlarged coverage of the confidence interval). We suspect that this small difference, which causes our demographic estimates to appear slightly conservative, probably occurs because we must calculate likelihoods at points along a grid of parameter values, rather than inferring them freely across the entire likelihood surface. Although decreasing the grid spacing (say, to either a $100 \times 100 \times 100$ grid, or the point where we could return the complete likelihood surface) would then systematically reduce our observed type I error rate, this option is not currently computationally feasible. The existing likelihood surface based on a $10 \times 10 \times 10$ grid required approximately three thousand CPU hours to calculate on a fast distributed-computing system. In comparison, a larger $100 \times 100 \times 100$ grid would take an impossible three million CPU hours to compute (equivalent to 343 years running on a single computer). We note, however, that the algorithm applied here varies only very slightly from expectations, and furthermore, because these small errors are conservative, they do not materially affect any of our main conclusions.

\section{Discussion}

We set out to determine whether Mexican free-tailed bat populations have experienced population growth and, if so, whether their onset of growth was concurrent with the expansion of human agricultural activity. The approximate Maximum Likelihood method applied here is a flexible and powerful analytical tool for testing hypotheses about historical demography. Similar methods have been applied previously to demographic analyses of human populations [22,25,26], and have relied on large datasets to infer complex demographic models [27]. Here, we employ these methods to address a specific aspect of the historical demography of Mexican freetailed bats, namely, the time of onset of population growth. Using coalescent-based inferential statistics, we directly address our hypothesis while ensuring that our data have sufficient power. Further, we address our hypothesis using amounts of sequence data that are typically available for many non-model organisms (only $\sim 1.2 \mathrm{~kb}$ ). We find that these bat populations have grown, but that their population growth began long before the arrival of humans in the Americas.

Several key points emerge from our analyses. First, a scenario whereby Mexican free-tailed bat populations are not growing (i.e., they are constant sized) is statistically unlikely. Considering the data points contained within the $95 \%$ confidence intervals for the demographic parameters (Additional file 3), modern effective sizes were always estimated to be larger than ancestral effective sizes (i.e., $N_{O} \neq N_{A}$ ). We therefore have statistical confidence that Mexican free-tailed bat populations have increased in size. Second, growth rates are not particularly large. The MLE of the combined likelihood surface suggests $\sim 48$-fold growth from the ancestral to modern effective population size (range: 16- to 324-fold growth). This rate of increase (MLE $\alpha=7 \times 10^{-5} /$ generation) is equivalent to a doubling of the bat population approximately every $31 \mathrm{kyr}$ (or $\sim 7,725$ generations). Such values do not suggest the extremely rapid growth that would be expected if Mexican free-tailed bat populations expanded from a small population size to their current numbers in response to human agricultural 
activity during the last few hundred years. Third, although we have little statistical power to place an upper bound on the time of onset of growth, our analysis has considerable power to infer the lower bound (cf. Additional file 4). Our MLE suggests that population growth in Mexican free-tailed bats began $\sim 230 \mathrm{ka}$, and the $95 \%$ confidence interval indicates a time for the onset of growth no younger than $\sim 120$ kya (or $~ 30,000$ generations). Because we infer such old times for the onset of growth in these bat populations, anthropogenic causes, which began no earlier than 9-15 kya [20,21], are statistically highly unlikely.

Several factors might confound our analysis, although none of these materially affect our main conclusions. First, coalescent analyses assume selective neutrality. There is no evidence for balancing selection (or equivalently, population structure) in our dataset; in such cases, Tajima's $D$ trends towards positive values. Positive, directional selection could produce the negative values of Tajima's $D$ that we observe, as well as elevate the number of singleton polymorphisms. However, levels of genetic diversity for our loci (e.g., Watterson's theta $\theta_{\mathrm{W}}$, or the number of segregating sites) are not consistent with positive, directional selection, which tends to considerably reduce genetic diversity [28]. Studies in other bat species also fail to reveal consistent evidence of positive selection at these two loci [29-31]. Finally, we note that strong positive, directional selection would lead us to infer high rates of growth and a very recent time of onset - the opposite of what we find here.

Second, both visual inspection of the sequence alignment and tests of the four-gamete rule [32] indicate that the mtDNA locus (and to a lesser extent, RAG2) have been affected by homoplasy (or alternately, recombination). Because it recreates polymorphism that is already present in the population, homoplasy tends to reduce the number of segregating sites and singleton polymorphisms that can actually be identified. However, the mutation rates of the two loci studied here - on the order of $10^{-8}$ and $10^{-10}$ - are sufficiently small that homoplasy should have only minor effects on either dataset, and consequently, on the demographic parameters that we infer.

Third, we may have over- or underestimated generation times. If the average generation interval is actually larger than we assume (i.e., $g>4$ years), then the time of onset of growth will be proportionally and linearly older than the values we report. Conversely, if the average generation time is less (i.e., $g<4$ years), then the time of onset of growth will be proportionally and linearly younger. However, even if the average generation time were 1 year, which is not supported by demographic studies in this bat species, our analyses would infer growth beginning no earlier than $\sim 30$ kya. This date still long precedes the arrival of humans in the Americas, let alone the emergence of widescale agriculture in North America.

\section{Conclusions}

Our analyses firmly support population growth in Mexican free-tailed bats, but reject a direct co-evolutionary connection with the development of human agriculture. We are then left asking what may have caused this increase in Mexican free-tailed bat numbers. The question is complicated by the fact that our data lack sufficient power to place an upper bound on the time of the onset of growth. However, since we are able to confidently infer a lower bound for this parameter (i.e., growth was no more recent than $\sim 120 \mathrm{ka}$ ), we find it likely that the signals of population growth observed in our data may be attributable to range expansion out of Pleistocene refugia. T. b. mexicana is a subtropical species, and typically migrates south to overwinter in Mexico (although some populations hibernate in coastal areas; [12]). In glacial periods during the Pleistocene, its range would have been restricted to Central America and Mexico, while interglacial periods would have seen a substantial range expansion and, we expect, concomitant population growth.

Finally, we cannot completely exclude the possibility that growth rates of Mexican free-tailed bat populations may have increased (or indeed decreased) relative to previous levels in response to extremely recent human activity, such as the development of large wind farms or the advent of wide-scale industrialized agriculture following the Second World War. A very recent uptick in the rate of growth on the background of a population that is already growing is extremely difficult to detect [22]. Similarly, it is also difficult to detect very recent decreases in effective population size. With only two genetic loci, we lack sufficient power to detect extremely recent deviations of this nature. Simulation studies indicate that hundreds to thousands of independent loci may be necessary to detect such recent events using sequence data [33]. Identifying very recent changes in population growth is difficult largely because the rate at which such demographic changes are recorded is constrained by low rates of mutation. It remains possible that such recent timescales might be more accessible by studying rapidly evolving microsatellites. Nevertheless, two key points are clear from our analyses: i) Mexican free-tailed bat populations have grown substantially in the past, and ii) this growth began well before humans arrived in the Americas. Given current evidence, it seems most parsimonious to assume that human agricultural activities have not driven this growth process to any major extent. 


\section{Methods}

\section{Samples and sequences}

Mexican free-tailed bats $\left(N_{S}=94\right)$ were sampled from 11 locations throughout Mexico and the southwestern United States (see [23] for locality information). Sample collection protocols were approved by the University of Tennessee Institutional Animal Care and Use Committee (UTK IACUC Protocol \#890). Two genetic loci were sequenced in these individuals: a haploid locus, the mitochondrial control region; and a diploid autosomal locus, the Recombination Activating Gene 2 (RAG2). We sequenced $474 \mathrm{bp}$ of the mitochondrial DNA (mtDNA) locus in 94 individuals (see methods in [23]), and 686 bp of the RAG2 gene (human genome homolog RAG2; chr11:36,613,495-36,619,812 in the February 2009 build of the human genome reference, hg19) in 75 individuals (i.e., 150 diploid chromosome copies). The $R A G 2$ region was sequenced using primers $179 \mathrm{~F}$ and 968R [34]. PCR was carried out in $12.5 \mu \mathrm{L}$ volumes containing $20 \mathrm{ng}$ genomic DNA, $1 \times$ PCR Gold buffer (Applied Biosystems), $2.5 \mathrm{mM} \mathrm{MgCl}_{2}, 0.8 \mathrm{mM}$ dNTP Blend (Applied Biosystems) $2.5 \mathrm{ng}$ each primer (Integrated DNA Technologies), and 0.0625 U AmpliTaq Gold DNA polymerase (Applied Biosystems). The PCR amplification profile consisted of initial hot start denaturation for $10 \mathrm{~min}$ at $95^{\circ} \mathrm{C}$, followed by 35 cycles of denaturation at $95^{\circ} \mathrm{C}$ for $30 \mathrm{~s}$, annealing at $55^{\circ} \mathrm{C}$ for 30 $\mathrm{s}$, and elongation at $72^{\circ} \mathrm{C}$ for $1 \mathrm{~min}$, with a final extension at $72^{\circ} \mathrm{C}$ for $10 \mathrm{~min}$.

Diploid RAG2 sequences were phased into haplotypes using a combination of computational and experimental methods. Fifteen individuals were either homozygous, or heterozygous at a single site, thereby allowing alleles at the RAG2 locus to be determined unambiguously. Uncertain diploid sequences were phased computationally using PHASE version 2.1 [35] with five replicate runs of a 2,000 step Markov chain, a burn-in of 1,000 steps, and a thinning interval of 2 . We were able to phase 43 individuals computationally using an output probability threshold of $95 \%$. Another 17 individuals, for which haplotypes were more ambiguous when subjected to computational phasing, were phased experimentally using standard cloning techniques. We first incubated $5 \mu \mathrm{L}$ of PCR product with $1 \mathrm{U}$ GoTaq Flexi polymerase (Promega) at $72^{\circ} \mathrm{C}$ for $10 \mathrm{~min}$ to add 3' dATPs for the TA cloning protocol. One $\mu \mathrm{L}$ of this reaction product was used for TOPO TA cloning (Invitrogen), from which plasmids were harvested from 3-12 E. coli colonies using the FastPlasmid Mini Purification System (5 Prime). Plasmids were sequenced using the BigDye v. 3.1 Terminator Cycle Sequencing Kit (Applied Biosystems) and the M13R primer (5'-CAGGAAACAGCTATGAC-3') on an ABI 3100 automated sequencer (Applied Biosystems). All sequences were edited and aligned using Sequencher v. 4.5 (GeneCodes). Sequence data are available online (GenBank: AY348008-AY348101, HM592833-HM592982).

\section{Summary statistics}

A suite of summary statistics was assembled to describe patterns of variation at each locus. Watterson's theta $\theta_{W}$, which is calculated from the number of segregating sites $S$, summarizes the total length of the genealogy [36], while the average number of pairwise differences $\theta_{\pi}$ summarizes the average coalescent time [37]. Both summary statistics are unbiased moment estimators of the population mutation rate $\left(\theta=s N_{e} \mu\right)$, where $s$ is the genomic scalar ( $s=1$ for uniparentally-inherited haploid loci, $s=4$ for biparentally-inherited autosomal loci), $N_{e}$ is the effective population size, and $\mu$ is the mutation rate. Tajima's $D$ [38] is the normalized difference between these two estimators, $\theta_{\pi^{-}}-\theta_{W}$, and has an expectation of zero under the standard neutral model. Nonzero values indicate deviations from this model. One such deviation is population growth, which tends to increase the number of singletons $\eta_{1}$ present in a sample (i.e., derived polymorphisms identified in only one individual). Because singletons elevate $\theta_{W}$ relative to $\theta_{\pi}$, growing populations are often characterized by negative values of Tajima's $D$. Similarly, an increased number of singletons also tends to elevate the number of unique haplotypes $h$. Therefore, all of these summaries were calculated for observed and simulated data using the $\mathrm{C}+$ + library libsequence [39].

The RAG2 mutation rate of $8.6 \times 10^{-10}$ substitutions/ site/year was estimated by comparing data for T. b. mexicana with an outgroup sequence from Eumops auripendulus (GenBank: AY834668) and assuming a divergence time of 22 mya [40]. The control region mutation rate was taken from published estimates for $T$. $b$. mexicana $\left(2.0 \times 10^{-8}\right.$ substitutions/site/year; [23]). Although the generation time of $T . b$. mexicana is not known with certainty, individuals reach sexual maturity at $\sim 1$ year, and have been known to live for at least 8 years in nature [41]. In our analyses, we initially assume a mean generation interval of 4 years (specifically, the average age of parents across all offspring, rather than the age at which parents first reproduce). The recombination rate for $R A G 2$ was inferred directly from the autosomal sequence data using PHASE [42].

\section{Population growth model}

To provide more nuanced inference than is possible from simple observation of summary statistics, we compared the data to a two-phase population growth model (Figure 1). Under this model, populations experience an early period of constant size (Phase 1 ), followed by a period of exponential growth (Phase 2). This model has 
three parameters: the ancestral population size $N_{A}$, the modern population size $N_{O}$, and the time of onset of growth $\tau$. Hence, considered in a backwards-in-time framework, the population size at any time $t$ is provided by

$$
N(t)= \begin{cases}N_{A}, & t>\tau \\ N_{A} e^{\alpha \tau}, & 0 \leq t \leq \tau\end{cases}
$$

where $\alpha$ is the exponential growth rate. Note that the scenario of constant effective size (i.e., $N_{O}=N_{A}$ ) is nested within this growth model (i.e., $\alpha=0$ ). Previous analyses of this subspecies are consistent with panmixia $[23,43]$, so we do not consider the effects of population structure in our model.

\section{Demographic inference}

To infer demographic parameters under the two-phase population growth model, we employed an inference procedure based on the Maximum Likelihood framework. Likelihood functions for complex demographic histories are too intractable to be derived analytically, and we therefore determine likelihoods by simulation with the $n$-coalescent under the infinite sites model [44]. Our approach thus belongs to a class of approximate methods [27]. Likelihoods are estimated from three summary statistics calculated from the data: the number of segregating sites $S$, which controls for the population mutation rate $\theta$; and the number of singletons $\eta_{1}$ and haplotypes $h$, both of which vary proportionally with population growth. All three summary statistics have the added convenience that they increase in integer units (i.e., $S, \eta_{1}, h \in \mathbb{N}$ ), which significantly raises the number of instances where the values of these summary statistics are identical in both observed and simulated datasets (see below). This characteristic greatly simplifies the calculation of likelihoods.

To explain the method simply, we aim to estimate the likelihood of a set of observed summary statistics $\varphi=$ $\left\{S, \eta_{1}, h\right\}$ across the parameter space of the two-phase growth model $\Lambda=\left\{N_{A}, N_{0}, \tau\right\}$. If the likelihood surface is known, it is a relatively trivial matter to determine the set of demographic parameters $\hat{\lambda}$ that maximizes the likelihood of the observed set of summary statistics $\varphi$. (This is the maximum likelihood estimate, or MLE, of $N_{A}, N_{O}$ and $\tau$ ). In practice, however, determining the complete likelihood surface is both difficult and computationally expensive. To generate an approximate alternative, we set initial bounds on the parameter space $A$ such that $N_{A} \in\left\{10^{4}, 10^{6}\right\}, N_{o} \in\left\{10^{4}, 5 \times 10^{7}\right\}$, and $\tau \in$ $\left\{0,5 \times 10^{5}\right\}$. We produced a uniform $10 \times 10 \times 10$ grid across this 3 -dimensional parameter space, although to ensure coalescence, we constrained the parameter space such that $N_{O} \geq N_{A}$ (or equivalently, $\alpha \geq 0$ ). In comparison, $\tau$ was allowed to vary freely across its full range.
At each point in the grid, we sampled $10^{6}$ coalescent trees for the haploid locus and $10^{6}$ ancestral recombination graphs (ARGs) for the autosomal locus using the simulation software $m s$ [45]. These simulations were conditioned on observed sequence lengths, inferred mutation rates, and for the autosomal locus, the inferred recombination rate. Instances matching the observed values of $S, \eta_{1}$, and $h$ (i.e., the likelihoods of $S, \eta_{1}$ and h) were counted directly from these simulated distributions. A combined likelihood surface was then constructed by multiplying likelihoods at each grid point across all loci $i$ and all summary statistics $j$

$$
L(\lambda)=\prod_{i} \prod_{j} f_{\lambda}\left(\phi_{i j}\right)
$$

where $f_{\lambda}$ is the observed marginal coalescent likelihood of $\varphi_{i j}$. The MLE is simply the set of demographic parameters $\hat{\lambda}=\left\{\hat{N}_{A}, \hat{N}_{0}, \hat{\tau}\right\}$ that maximizes $L(\lambda)$. Although calculating the joint coalescent likelihood of $\varphi_{i j}$ would be preferable, this would require orders of magnitude more computational time than the method applied here and is not currently computationally feasible. Validation simulations (see below) show that using marginal values does not affect the accuracy of our method.

As is traditional, we report the natural log of likelihoods in preference to the likelihoods themselves; that is, values are reported on the scale $(-\infty, 0)$ rather than $(0,1)$. Confidence intervals were constructed using standard methods. In brief, confidence intervals incorporate all values $x$ such that

$$
x \in\left\{\lambda: \ln L(\lambda) \geq \ln L(\hat{\lambda})-\frac{\chi_{d f}^{2}}{2}\right\}
$$

Because we consider a parameter space with three independent demographic parameters, the quartile of the $\chi^{2}$ distribution with 3 degrees of freedom and a one-tailed probability of 0.05 was applied. Profile likelihoods were also calculated using standard methods; however, because these confidence intervals represent only a single dimension (i.e., each demographic parameter is considered separately), we applied a $\chi^{2}$ distribution with only 1 degree of freedom.

\section{Validation}

Our inference method was validated by generating $10^{3}$ coalescent trees and ARGs using values of $N_{A}, N_{O}$ and $\tau$ chosen randomly from a uniform distribution across the parameter space. We applied the approximate Maximum Likelihood method described above to the values of $S, \eta_{1}$ and $h$ returned by each simulation. Instances where the known (i.e., simulated) values of $N_{A}, N_{O}$ and $\tau$ were included within the confidence intervals of the 
inferred demography were considered successful. Deviation from expectation is reported as a type I error rate.

\section{Additional material}

\section{Additional file 1: Log-likelihood surface $\left(N_{A}\right.$ versus $\left.\tau\right)$ for the} haploid mtDNA control region

Additional file 2: Log-likelihood surface $\left(N_{A}\right.$ versus $\left.\tau\right)$ for the autosomal RAG2 locus

Additional file 3: Grid points forming the $95 \%$ confidence interval of the three-dimensional parameter space ranked by likelihood value.

Additional file 4: Profile likelihood curves drawn from the combined likelihood surface for the haploid mtDNA control region and autosomal RAG2 locus.

\section{Acknowledgements}

Funding for this study was provided by the Department of Ecology and Evolutionary Biology of the University of Tennessee, Bat Conservation International, Sigma Xi, and the American Museum of Natural History. We thank Arizona Research Laboratories at the University of Arizona for computational support, Grand Valley State University for logistical support, and Liliana Dávalos for critical comments on the manuscript.

\section{Author details}

'Department of Biology, Grand Valley State University, Allendale, MI 49401, USA. ${ }^{2}$ Institute of Molecular BioSciences, Massey University, Palmerston North 4442, New Zealand. ${ }^{3}$ Allan Wilson Centre for Molecular Ecology and Evolution, Palmerston North, New Zealand. ${ }^{4}$ Bio-Protection Research Centre, Canterbury, New Zealand. ${ }^{5}$ Department of Ecology and Evolutionary Biology, University of Tennessee, Knoxville, TN 37996, USA.

\section{Authors' contributions}

$A L R, M P C$, and GFM conceived of the study and participated in its design. $A L R$ and $V A B$ carried out the molecular genetic studies. ALR aligned the sequence data and performed initial statistical analyses. MPC wrote code for and performed the approximate Maximum Likelihood analyses. ALR and MPC drafted the manuscript. All authors read and approved the final manuscript.

Received: 12 November 2010 Accepted: 1 April 2011

Published: 1 April 2011

\section{References}

1. Currey RJC, Dawson SM, Slooten E, Schneider K, Lusseau D, Boisseau OJ, Haase P, Williams JA: Survival rates for a declining population of bottlenose dolphins in Doubtful Sound, New Zealand: an information theoretic approach to assessing the role of human impacts. Aquat Conserv 2009, 19:658-670.

2. Stallings CD: Fishery-independent data reveal negative effect of human population density on Caribbean predatory fish communities. PLoS One 2009, 4:e5333.

3. Regular PM, Robertson GJ, Montevecchi WA, Shuhood F, Power T, Ballam D, Piatt JF: Relative importance of human activities and climate driving common murre population trends in the Northwest Atlantic. Polar Biol 2010, 33:1215-1226.

4. May GE, Gelembiuk GW, Panov VE, Orlova MI, Lee CE: Molecular ecology of zebra mussel invasions. Mol Ecol 2006, 15:1021-1031.

5. Hulva P, Fornusková A, Chudárková A, Evin A, Allegrini B, Benda P, Bryja J: Mechanisms of radiation in a bat group from the genus Pipistrellus inferred by phylogeography, demography and population genetics. $\mathrm{Mol}$ Ecol 2010, 19:5417-5431.

6. Purcell JE, Uye S, Lo WT: Anthropogenic causes of jellyfish blooms and their direct consequences for humans: a review. Mar Ecol Prog Ser 2007, 350:153-174
7. Hugo S, van Rensburg BJ: The maintenance of a positive spatial correlation between South African bird species richness and human population density. Global Ecol Biogeogr 2008, 17:611-621.

8. Goossens B, Chikhi L, Ancrenaz M, Lackman-Ancrenaz I, Andau P, Bruford MW: Genetic signature of anthropogenic population collapse in orang-utans. PLOS Biol 2006, 4:e25.

9. Liu Z, Ren B, Wu R, Zhao L, Hao Y, Wang B, Wei F, Long Y, Li M: The effect of landscape features on population genetic structure in Yunnan snubnosed monkeys (Rhinopithecus bieti) implies an anthropogenic genetic discontinuity. Mol Ecol 2009, 18:3831-3846.

10. Funk WC, Forsman ED, Johnson M, Mullins TD, Haig SM: Evidence for recent population bottlenecks in northern spotted owls (Strix occidentalis caurina). Conserv Genet 2010, 11:1013-1021.

11. Davis RB, Herreid CF, Short HL: Mexican free-tailed bats in Texas. Ecol Monogr 1962, 32:311-346

12. Cockrum EL: Migration in the guano bat, Tadarida brasiliensis. Misc Pub Univ Kansas Mus Nat Hist 1969, 51:303-336.

13. Betke M, Hirsh DE, Makris NC, McCracken GF, Procopio M, Hristov NI, Tang S, Bagchi A, Reichard JD, Horn JW, Crampton S, Cleveland CJ, Kunz TH: Thermal imaging reveals significantly smaller Brazilian freetailed bat colonies than previously estimated. J Mammal 2008, 89:18-24

14. Kunz TH, Whitaker JO, Wadanoli MD: Dietary energetics of the insectivorous Mexican free-tailed bat (Tadarida brasiliensis) during pregnancy and lactation. Oecologia 1995, 101:407-415.

15. Gatehouse AG: Behavior and ecological genetics of wind-borne migration by insects. Annu Rev Entomol 1997, 42:475-502.

16. McCracken GF, Gillam EH, Westbrook JK, Lee YF, Jensen ML, Balsley BB: Brazilian free-tailed bats (Tadarida brasiliensis: Molossidae, Chiroptera) at high altitude: links to migratory insect populations. Integr Comp Biol 2008, 48:107-118.

17. McCracken GF, Brown VA, Eldridge M, Federico P, Westbrook JK Opportunistic predation by bats tracks and exploits changes in insect pest populations: evidence from quantitative ( $q P C R$ ) analysis of fecal DNA. Bat Res News 2008, 49:147.

18. Lee YF, McCracken GF: Dietary variation of Brazilian free-tailed bats link to migratory populations of pest insects. J Mammal 2005, 86:67-76.

19. Cleveland CJ, Betke M, Federico P, Frank JD, Hallam TG, Horn J, López JD, McCracken GF, Medellín RA, Moreno-Valdez A, Sansone CG, Westbrook JK, Kunz TH: Economic value of the pest control service provided by Brazilian free-tailed bats in south-central Texas. Front Ecol Environ 2006, 4:238-243.

20. Piperno DR, Ranere AJ, Holst I, Iriarte J, Dickau R: Starch grain and phytolith evidence for early ninth millennium B.P. maize from the Central Balsas River Valley, Mexico. Proc Natl Acad Sci USA 2009, 106:5019-5024.

21. Gilbert MTP, Jenkins DL, Götherström A, Naveran N, Sanchez JJ, Hofreiter M, Thomsen PF, Binladen J, Higham TFG, Yohe RM, Parr R, Cummings LS, Willerslev E: DNA from pre-Clovis human coprolites in Oregon, North America. Science 2008, 320:786-789.

22. Cox MP, Morales DA, Woerner AE, Sozanski J, Wall JD, Hammer MF: Autosomal resequence data reveal late Stone Age signals of population expansion in sub-Saharan African foraging and farming populations. PLoS One 2009, 4:e6366.

23. Russell AL, Medellín RA, McCracken GF: Genetic variation and migration in the Mexican free-tailed bat (Tadarida brasiliensis mexicana). Mol Ecol 2005, 14:2207-2222.

24. Metni Pilkington M, Wilder JA, Mendez FL, Cox MP, Woerner A, Angui T, Kingan S, Mobasher Z, Batini C, Destro-Bisol G, Soodyall H, Strassmann Bl, Hammer MF: Contrasting signatures of population growth for mitochondrial DNA and $\mathrm{Y}$ chromosomes among human populations in Africa. Mol Biol Evol 2008, 25:517-525.

25. Pluzhnikov A, Di Rienzo A, Hudson RR: Inferences about human demography based on multilocus analyses of noncoding sequences. Genetics 2002, 161:1209-1218

26. Voight BF, Adams AM, Frisse LA, Qian Y, Hudson RR, Di Rienzo A: Interrogating multiple aspects of variation in a full resequencing data set to infer human population size changes. Proc Natl Acad Sci USA 2005, 102:18508-18513.

27. Plagnol V, Wall JD: Possible ancestral structure in human populations. PLOS Genet 2006, 2:e105. 
28. Peng Y, Shi H, Qi XB, Xiao CJ, Zhong H, Ma RL, Su B: The ADH1B Arg47His polymorphism in East Asian populations and expansion of rice domestication in history. BMC Evol Biol 2010, 10:15.

29. Lamb JM, Ralph TMC, Goodman SM, Bogdanowicz W, Fahr J, Gajewska M, Bates PJJ, Eger J, Benda P, Taylor PJ: Phylogeography and predicted distribution of African-Arabian and Malagasy populations of giant mastiff bats, Otomops spp. (Chiroptera: Molossidae). Acta Chiropterol 2008, 10:21-40

30. Juste J, Bilgin R, Muñoz J, Ibáñez C: Mitochondrial DNA signatures at different spatial scales: from the effects of the Straits of Gibraltar to population structure in the meridional serotine bat (Eptesicus isabellinus). Heredity 2009, 103:178-187.

31. Martins FM, Templeton AR, Pavan ACO, Kohlbach BC, Morgante JS: Phylogeography of the common vampire bat (Desmodus rotundus): marked population structure, Neotropical Pleistocene vicariance and incongruence between nuclear and mtDNA markers. BMC Evol Biol 2010 9:294

32. Woerner AE, Cox MP, Hammer MF: Recombination-filtered genomic datasets by information maximization. Bioinformatics 2007, 23:1851-1853.

33. Adams AM, Hudson RR: Maximum-likelihood estimation of demographic parameters using the frequency spectrum of unlinked single-nucleotide polymorphisms. Genetics 2004, 168:1699-1712

34. Stadelmann B, Lin LK, Kunz TH, Ruedi M: Molecular phylogeny of New World Myotis (Chiroptera, Vespertilionidae) inferred from mitochondrial and nuclear DNA genes. Mol Phylogenet Evol 2007, 43:32-48.

35. Stephens M, Smith NJ, Donnelly P: A new statistical method for haplotype reconstruction from population data. Am J Hum Genet 2001, 68:978-989.

36. Watterson GA: On the number of segregating sites in genetical models without recombination. Theor Popul Biol 1975, 7:256-276.

37. Tajima F: Evolutionary relationship of DNA sequences in finite populations. Genetics 1983, 105:437-460.

38. Tajima F: Statistical method for testing the neutral mutation hypothesis by DNA polymorphism. Genetics 1989, 123:585-595.

39. Thornton K: libsequence: a C++ class library for evolutionary genetic analysis. Bioinformatics 2003, 19:2325-2327.

40. Teeling EC, Springer MS, Madsen O, Bates P, O'Brien SJ, Murphy WJ: A molecular phylogeny for bats illuminates biogeography and the fossi record. Science 2005, 307:580-584.

41. Brunet-Rossinni A, Austad S: Ageing studies on bats: a review. Biogerontol 2004, 5:211-222

42. Li N, Stephens M: Modelling linkage disequilibrium, and identifying recombination hotspots using SNP data. Genetics 2003, 165:2213-2233.

43. McCracken GF, McCracken MK, Vawter AT: Genetic structure in migratory populations of the bat Tadarida brasiliensis mexicana. J Mammal 1994, 75:500-514.

44. Wakeley J: Coalescent Theory: An Introduction Greenwood Village: Roberts \& Company Publishers; 2008

45. Hudson RR: Generating samples under a Wright-Fisher neutral model of genetic variation. Bioinformatics 2002, 18:337-338.

doi:10.1186/1471-2148-11-88

Cite this article as: Russell et al:: Population growth of Mexican freetailed bats (Tadarida brasiliensis mexicana) predates human agricultural activity. BMC Evolutionary Biology 2011 11:88.

\section{Submit your next manuscript to BioMed Central and take full advantage of:}

- Convenient online submission

- Thorough peer review

- No space constraints or color figure charges

- Immediate publication on acceptance

- Inclusion in PubMed, CAS, Scopus and Google Scholar

- Research which is freely available for redistribution

Submit your manuscript at www.biomedcentral.com/submit
C Biomed Central 